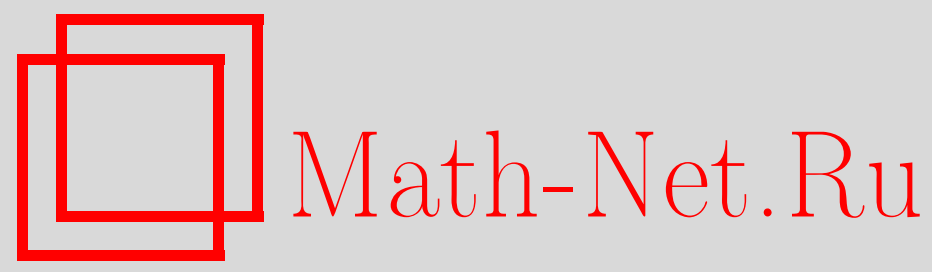

А. В. Покровский, Устранимые особенности решений дивергентных эллиптических уравнений второго порядка, Матем. заметки, 2005, том 77, выпуск 3, 424-433

DOI: https://doi.org/10.4213/mzm2503

Использование Общероссийского математического портала Math-Net.Ru подразумевает, что вы прочитали и согласны с пользовательским соглашением http://www . mathnet.ru/rus/agreement

Параметры загрузки:

IP: 54.84 .234 .179

26 апреля 2023 г., $16: 31: 37$

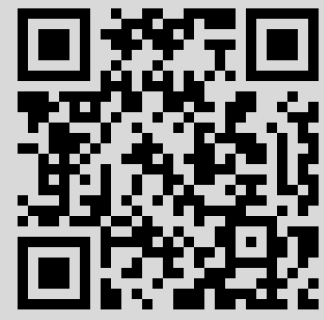




\section{УСТРАНИМЫЕ ОСОБЕННОСТИ РЕШЕНИЙ ДИВЕРГЕНТНЫХ ЭЛЛИПТИЧЕСКИХ УРАВНЕНИЙ ВТОРОГО ПОРЯДКА}

\section{А. В. Покровский}

Пусть $L$ - равномерно эллиптический линейный дифференциальный оператор второго порядка в дивергентной форме с ограниченными и измеримыми коэффициентами в ограниченной области $G \subset \mathbb{R}^{n}(n \geqslant 2)$. В работе вводятся подклассы соболевского класса $W^{1,2}(G)_{\text {loc }}$, содержащие обобщенные решения уравнения $L u=0$, в которых замкнутые множества неизолированных особых точек, устранимые для таких решений, полностью описываются в терминах хаусдорфовых мер.

Библиографоия: 35 названий.

Пусть $G$ - область в $\mathbb{R}^{n}, n \geqslant 2$,

$$
L f=\sum_{i, j=1}^{n} D_{i}\left(a_{i j}(x) D_{j} f\right)+\sum_{i=1}^{n} D_{i}\left(b_{i}(x) f\right)+\sum_{i=1}^{n} c_{i}(x) D_{i} f+d(x) f
$$

$x=\left(x_{1}, \ldots, x_{n}\right) \in G, D_{i}=\partial / \partial x_{i}, a_{i j}(x)=a_{j i}(x), i, j=1, \ldots, n-$ линейный дифференциальньй оператор второго порядка с ограниченньпи и измеримыми действительньми коэффициентами в этой области,

$$
\left|a_{i j}(x)\right| \leqslant M, \quad\left|b_{i}(x)\right| \leqslant M, \quad\left|c_{i}(x)\right| \leqslant M, \quad|d(x)| \leqslant M
$$

для некоторого $M>0$ и почти всех $x \in G, i, j=1, \ldots, n$, и $L$ равномерно эллиптичен в $G$, т.е. существует такое $\lambda>0$, что при всех $\xi \in \mathbb{R}^{n}$ и $x \in G$ выполняется неравенство

$$
\sum_{i, j=1}^{n} a_{i j}(x) \xi_{i} \xi_{j} \geqslant \lambda|\xi|^{2}=\lambda\left(\xi_{1}^{2}+\cdots+\xi_{n}^{2}\right)
$$

Пусть $1 \leqslant p<\infty$. Как обычно, $L_{p}(G)$ - пространство всех вещественных измеримых в области $G$ функций $f$, суммируемых в степени $p$, с нормой

$$
\left\|f \mid L_{p}(G)\right\|:=\left(\int_{G}|f(x)|^{p} d x\right)^{1 / p}
$$

Эта работа выполнена при частичной поддержке фонда INTAS, грант № 9900089, и Государственной программы Украины, грант № $0102 \mathrm{U} 000917$. 
$W^{1, p}(G)$ - соболевскоепространство всех вещественных измеримых в $G$ функций $f$, имеющих обобщенные производные $D_{i} f, i=1, \ldots, n$, для которых конечна норма

$$
\left\|f \mid W^{1, p}(G)\right\|:=\left(\int_{G}|f(x)|^{p} d x\right)^{1 / p}+\sum_{i=1}^{n}\left(\int_{G}\left|D_{i} f(x)\right|^{p} d x\right)^{1 / p}
$$

$W_{0}^{1, p}(G)$ - замыкание пространства $C_{0}^{\infty}(G)$ всех вещественных финитных бесконечно дифференцируемых функций с компактньм носителем в области $G$ по норме пространства $W^{1, p}(G)$. Если $0<\alpha \leqslant 1$, то, как обычно, $C^{\alpha}(G)$ - множество всех вещественных непрерьвных в $G$ функций $f$, удовлетворяющих там условию Гёльдера с показателем $\alpha$, $C^{1, \alpha}(G)$ - множество всех вещественных функций $f$, непрерывно дифференцируемых в $G$, все первые частные производные которых удовлетворяют условию Гёльдера с показателем $\alpha$ в области $G$. Если в каждой области $G \subset \mathbb{R}^{n}$ определен некоторьй функциональный класс $H(G)$, так что для каждой пары областей $G_{1}, G_{2} \subset \mathbb{R}^{n}$ из $G_{1} \subset G_{2}$

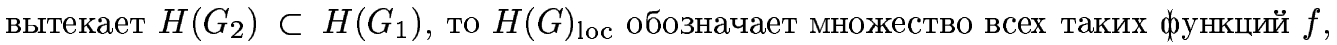
определенных в $G$, что $f \in H\left(G_{0}\right)$ для каждой подобласти $G_{0} \Subset G$. Символ $\nabla$ используется для обозначения градиента функции: $\nabla f=\left(D_{1} f, \ldots, D_{n} f\right)$.

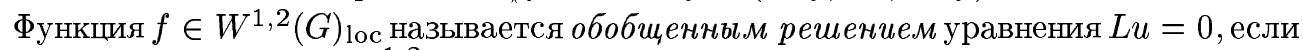
для любой функции $\phi \in W_{0}^{1,2}(G)$ вьполняется равенство

$$
\begin{aligned}
(L f, \phi):= & -\sum_{i, j=1}^{n} \int_{G} a_{i j}(x) D_{i} f(x) D_{j} \phi(x) d x-\sum_{i=1}^{n} \int_{G} b_{i}(x) f(x) D_{i} \phi(x) d x \\
& +\sum_{i=1}^{n} \int_{G} c_{i}(x) D_{i} f(x) \phi(x) d x+\int_{G} d(x) f(x) \phi(x) d x=0 .
\end{aligned}
$$

В этом случае из определения пространства $W_{0}^{1,2}(G)$ вытекает, что при $f \in W^{1,2}(G)$ равенство (2) выполняется для всех функций $\phi \in W_{0}^{1,2}(G)$.

Если $x \in \mathbb{R}^{n}$ и $r>0$, то $B(x, r)$ обозначает открытьй евклидов шар с центром в точке $x$ и радиусом $r>0: B(x, r):=\left\{y \in \mathbb{R}^{n}:|x-y|<r\right\}$.

Пусть $t_{0}>0, g(t)$ - положительная непрерывная неубьвающая функция, определенная при $0<t<t_{0}$, и пусть $E$ - множество в $\mathbb{R}^{n}$. Напомним, что (внешней) мерой $X а y с д о р ф а$ mes $_{g} E$ множества $E$ относительно измеряющей функции $g$ назьвается конечньй или равньй $+\infty$ предел при $t \rightarrow 0$ величины inf $\left(\sum_{i} g\left(r_{i}\right)\right)$, где инфимум берется по всем не более, чем счетным множествам открытых шаров $\left\{B\left(x_{i}, r_{i}\right)\right\}_{i}$ c $r_{i} \leqslant t$, образуюших покрытие множества $E$. Если $g(t)=t^{\gamma}, \gamma \geqslant 0$, то хаусдорфова мера множества $E$ относительно измеряющей функции $g$ называется длиной по Хаусдорфу порядка $\gamma$ множества $E$ и обозначается $\operatorname{mes}^{\gamma} E$.

Цель настоящей заметки состоит в обобщении следующей теоремы Е. П. Долженко об устранимых особенностях гармонических функций (т.е. решений уравнения $\Delta f=0$, где $\Delta=\partial^{2} / \partial x_{1}^{2}+\cdots+\partial^{2} / \partial x_{n}^{2}$-оператор Лапласа в $\left.\mathbb{R}^{n}\right)$ на случай обобщенных решений уравнения $L f=0$, где $L$ - произвольньй равномерно эллиптический оператор вида (1) с ограниченньми измеримыми коэффициентами.

ТЕОРЕМА ДолЖенко [1], [2]. Пусть $G$ - область в $\mathbb{R}^{n}, n \geqslant 2, E$ - замкнутое мнохество в этой области, $E \neq G$, и пусть $0<\alpha<1$. Тогда для устранимости мнохсества $E$ для гармонических функиий в классе $C^{1, \alpha}(G)_{\text {loc }}$ (т.е. для 


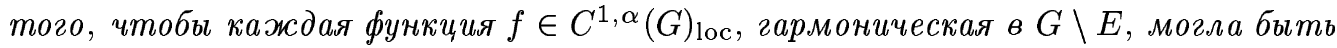
продолжена как гармоническая функиия $c G \backslash E$ на $G$ ) необходимо и достаточно, чтобы хаусдорфова длина множества $E$ порядка $n-1+\alpha$ равнялась нулю: mes $^{n-1+\alpha} E=0$.

По поводу других результатов об устранимых особенностях гармонических функций и решений линейных дифференциальных уравнений с достаточно гладкими коэффициентами см. работы [3]- [18]. Отметим, что в отличие от этих работ мы рассматриваем дифференциальные операторы без каких-либо априорных предположений о гладкости их коэффициентов (и, как следствие, - о гладкости их решений, см., например, [19, c. 160, 161]), и доказываемая ниже теорема 1 является, по-видимому, первым результатом, где устранимые особенности обобщенных решений обших дивергентных равномерно эллиптических линейньх уравнений второго порядка с ограниченными измеримьми коэффициентами полностью описаны в терминах хаусдорфовых мер.

Напомним, что по теореме Де Джорджи и Нэша [20], [21] (см. также [19], [22], [23]) каждое обобщенное решение уравнения $L u=0$ с точностью до переопределения на множестве нулевой меры является непрерывной функцией, которая на каждом компакте $K \Subset G$ удовлетворяет условию Гёльдера с некоторьм показателем $\alpha>0$, зависящем только от оператора $L$ и области $G$ (в дальнейшем, говоря об обобщенных решениях, мы всегда предполагаем такое переопределение выполненным). С другой стороны, для любого $\alpha>0$ можно привести пример оператора вида (1) c $G=\mathbb{R}^{n}$ и обобщенного решения в $\mathbb{R}^{n}$ уравнения $L u=0$, которое не удовлетворяет условию Гёльдера с показателем $\alpha$ ни в какой окрестности точки $x=0$ (см. [19, с. 133]). Эти результаты показьвают, что в случае произвольного равномерно эллиптического оператора $L$ вида (1) с ограниченньми измеримыми коэффициентами постановка задачи об устранимых особенностях

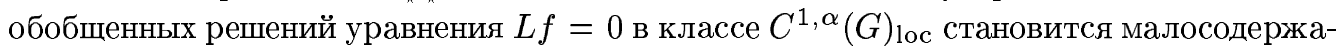
тельной: решения, вообще говоря, могут не принадлежать этому классу. Поэтому естественно возникает вопрос о том, в каких классах функций следует рассматривать задачу о стирании особенностей в этом случае, чтобы с одной стороны имело заключение типа вьшеприведенной теоремы Долженко, а с другой - каждое обобщенное решение рассматриваемого уравнения принадлежало бы такому классу. В настоящей работе дается одно из таких определений.

Всюду ниже вплоть до замечания 4 мы предполагаем выполненным следующее условие: для любой неотрицательной функции $\phi \in C_{0}^{\infty}(G)$ справедливо неравенство

$$
\int_{G}\left(d \phi-\sum_{i=1}^{n} b_{i} D_{i} \phi\right) d x \leqslant 0
$$

которое, в частности, имеет место, если $b_{i}=0$ и $d \leqslant 0$ п.в. в $G, i=1, \ldots, n$.

Пусть $x \in \mathbb{R}^{n}$ и $r>0, f \in W^{1,2}(B(x, r))$. Обозначим через $f_{L, x, r}$ обобщенное решение задачи Дирихле $L u=0$ в $B(x, r), u-f \in W_{0}^{1,2}(B(x, r))$, которое при условии (3) всегда существует и единственно (см. [23, теорема 8.3]).

Всюду ниже $h(t)$ - непрерьвная положительная и неубьвающая функция, определенная при $t>0, g(t):=t^{n-1} h(t)$.

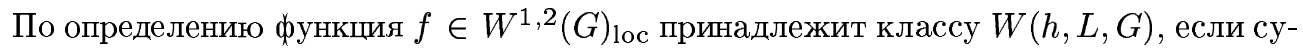
ществует такое $C \geqslant 0$, что для каждого шара $B(x, r)$, лежащего со своим замыканием в 
области $G$, выполняется неравенство

$$
\left\|f-f_{L, x, r} \mid W^{1,2}(B(x, r))\right\| \leqslant C r^{n / 2} h(r) .
$$

Если $h(t)=t^{\alpha}, \alpha \geqslant 0$, то $W(h, L, G)=: W^{\alpha}(L, G)$.

Пусть $x \in \mathbb{R}^{n}$ и $r>0$. Тогда для любой функции $\phi \in W_{0}^{1,2}(B(x, r))$ выполняется неравенство Пуанкаре

$$
\left\|\phi\left|L_{2}(B(x, r))\|\leqslant C r\| \nabla \phi\right| L_{2}(B(x, r))\right\|,
$$

где $C$ зависит только от размерности $n$ (см., например, [23, с. 161]). Из этого неравенства вытекает, что в определении классов $W(h, L, G)$ неравенство (4) можно заменить неравенством

$$
\left\|\nabla\left(f-f_{L, x, r}\right) \mid L_{2}(B(x, r))\right\| \leqslant C r^{n / 2} h(r) .
$$

Отметим, что идея такого определения функциональных классов восходит к работам В.С. Федорова по теории моногенности и представлению голоморфных функций одного комплексного переменного с компактным множеством особых точек в виде интеграла типа Коши. В этих работах условия на функцию давались в терминах ее локальных приближений в равномерной метрике или в среднем голоморфными функциями (см. [24]). В последствии эта идея широко использовалась в работах Б. Ж. Ищанова (cм. [8]-[10], [25]).

Пусть $E$ - замкнутое множество в области $G, E \neq G$.

ОПРЕДЕЛЕНИЕ 1. Множество $E$ называется $L$-устранимым в классе $W(h, L, G)$, если любая функция $f \in W(h, L, G)$, являющаяся обобщенным решением уравнения $L u=0$ на множестве $G \backslash E$ (т.е. удовлетворяющая равенству (2) для каждой вещественной бесконечно дифференцируемой функции $\phi$ с носителем, принадлежащем $G \backslash E$ ), является после, возможно, переопределения ее на множестве $E$, обобщенным решением уравнения $L u=0$ в области $G$.

Всюду далее предполагается, что область $G$ ограничена.

В принятых вьше обозначениях сфформулируем основной результат работы.

Tеорема 1. Для L-устранимости множества $E$ в классе $W(h, L, G)$ необходимо и достаточно, чтобы хаусдорфова мера $E$ относительно измеряющей функиии $g(t)=t^{n-1} h(t)$ равнялась нулю: mes $_{g} E=0$.

Для доказательства теоремы нам потребуются три хорошо известных результата, которые мы сформулируем в виде лемм.

Для формулировки первого из них напомним, что двоичным кубом в $\mathbb{R}^{n}$ назьвается всякий куб вида $\left[s_{1} 2^{-k},\left(s_{1}+1\right) 2^{-k}\right] \times \cdots \times\left[s_{n} 2^{-k},\left(s_{n}+1\right) 2^{-k}\right]$, где $s_{1}, \ldots, s_{n}$ - целые числа, $k$ - натуральное. Если $Q$ - двоичньй куб и $d>0$, то $d Q$ обозначает куб с тем же самым центром, что и $Q$, и длиной ребра в $d$ раз большей, чем длина ребра $Q$.

ЛЕмма 1 (Р. Харви и Дж. Полкинг [5], см. также [15, с. 20-22]). Пусть $\left\{Q_{l}\right\}_{l=1}^{m}$, $m \in \mathbb{N}$, - конечное множество двоичных кубов, внутренности которых попарно не пересекаются, $r_{l}$ - длина ребра $Q_{l}, l=1, \ldots, m$. Тогда существует такое множество функиий $\left\{\phi_{l}\right\}_{l=1}^{m} \subset C_{0}^{\infty}\left(\mathbb{R}^{n}\right)$, принимаюших значения только на интервале $[0,1]$, что носитель $\phi_{l}$ содержится в кубе $\frac{3}{2} Q_{l}$, кажсдая частная производная функиии $\phi_{l}$ порядка $k>0$ по модулю не превосходит $C(n, k) r_{l}^{-k}$, где величина $C(n, k)$ зависит лишь от $n$ и $k, i=1, \ldots, m$, и на множестве $\bigcup_{l=1}^{m} Q_{l}$ выполняется равенство $\sum_{l=1}^{m} \phi_{l}(x)=1$. 
ЛЕмма 2 (О. Фростман [26], см. также [4], [27]). Пусть $g(t)$ - положительная не-

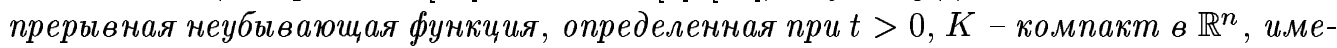
ющий положительную хаусдорфову меру относительно измеряющей функиии $g$. Тогда существует такая борелевская мера $\mu$ сносителем на $K$, что $\mu(K)>0 u$ $\mu(B(x, r)) \leqslant g(r)$ для кажсдого шара $B(x, r) \subset \mathbb{R}^{n}$.

Следующая лемма - частньй случай одного результата В. Г. Мазьи $[28$, с. 56,57$]$.

ЛЕмма 3. Пусть борелевская мера $\mu$ скомпактным носителем в $\mathbb{R}^{n}$ удовлетворяет условию

$$
c(\mu):=\sup \left\{r^{1-n} \mu(B(x, r)): x \in \mathbb{R}^{n}, r>0\right\}<\infty .
$$

Тогда для кажсдой функиии $\phi \in C_{0}^{\infty}\left(\mathbb{R}^{n}\right)$ выполняется неравенство

$$
\int_{\mathbb{R}^{n}}|\phi(x)| d \mu(x) \leqslant K c(\mu)\left\|\nabla \phi \mid L_{1}\left(\mathbb{R}^{n}\right)\right\|
$$

әде $K$ зависит только от размерности $n$.

Перейдем к ДОКАЗАТЕЛЬСТВУ ТЕОРЕМЫ, которое фактически получается последовательным применением трех вьшеприведенных лемм. Начнем с достаточности. Пусть $f$ - функция из класса $W(h, L, G)$, являющаяся обобщенным решением уравнения $L u=0$ на множестве $G \backslash E$. Зафиксируем произвольньм образом функцию $\phi \in C_{0}^{\infty}(G)$ и $\varepsilon>0$. Поскольку множество $E(\phi):=E \cap \operatorname{supp} \phi$ - компакт, a mes $g=0$, существует такое конечное множество двоичных кубов $\left\{Q_{l}\right\}_{l=1}^{m}, m \in \mathbb{N}$, внутренности которых попарно не пересекаются, что $E(\phi) \subset \bigcup_{l=1}^{m} Q_{i}$ и $\sum_{l=1}^{m} g\left(r_{l}\right) \leqslant \varepsilon$, где $r_{l}$ обозначает длину ребра куба $Q_{l}, l=1, \ldots, m$ (каждьй шар содержится в объединении не более чем $5^{n}$ двоичных кубов, длина ребра которых не превосходит радиуса этого шара). При этом, очевидно, можно предполагать, что $\bigcup_{l=1}^{m} B\left(x_{l}, \sqrt{n} r_{l}\right) \subset G$, где $x_{l}$ - центр куба $Q_{l}, l=1, \ldots, m$. Пусть $\left\{\phi_{l}\right\}_{l=1}^{m}$ - система функций, образующая разбиение единицы из леммы 1 для системы кубов $\left\{Q_{l}\right\}_{l=1}^{m}$. Пользуясь тем, что $f_{l}:=f_{L, x_{l}, \sqrt{n} r_{l}}$ обобщенное решение уравнения $L u=0$ внутри шара $B\left(x_{l}, \sqrt{n} r_{l}\right) \supset \frac{3}{2} Q_{l}$, оценкой производных функций $\phi_{l}$ из леммы 1 , определением класса $W(h, L, G)$ и неравенством Гёльдера, получаем

$$
\begin{aligned}
|(L f, \phi)|= & \left|\sum_{l=1}^{m}\left(L f, \phi \phi_{l}\right)+\left(L f, \phi\left(1-\sum_{l=1}^{m} \phi_{l}\right)\right)\right| \leqslant \sum_{l=1}^{m}\left|\left(L f-L f_{l}, \phi \phi_{l}\right)\right| \\
\leqslant & \sum_{l=1}^{m}\left(\left|\sum_{i, j=1}^{n} \int_{B\left(x_{l}, \sqrt{n} r_{l}\right)} a_{i j} D_{i}\left(f-f_{l}\right) D_{j}\left(\phi \phi_{l}\right) d x\right|\right. \\
& +\left|\sum_{i=1}^{n} \int_{B\left(x_{l}, \sqrt{n} r_{l}\right)} b_{i}\left(f-f_{l}\right) D_{i}\left(\phi \phi_{l}\right) d x\right| \\
& \left.+\left|\sum_{i=1}^{n} \int_{B\left(x_{l}, \sqrt{n} r_{l}\right)} c_{i} D_{i}\left(f-f_{l}\right)\left(\phi \phi_{l}\right) d x\right|+\left|\int_{B\left(x_{l}, \sqrt{n} r_{l}\right)} d\left(f-f_{l}\right)\left(\phi \phi_{l}\right) d x\right|\right) \\
\leqslant & \sum_{l=1}^{m} C(L, G, n, \phi)\left\|f-f_{l} \mid W^{1,2}\left(B\left(x_{l}, \sqrt{n} r_{l}\right)\right)\right\| r_{l}^{n / 2} r_{l}^{-1}
\end{aligned}
$$




$$
\begin{aligned}
& \leqslant \sum_{l=1}^{m} C(L, G, n, \phi, f, \alpha) r_{l}^{n / 2} h\left(r_{l}\right) r_{l}^{n / 2-1} \\
& =\sum_{l=1}^{m} C(L, G, n, \phi, f, \alpha) g\left(r_{l}\right) \leqslant C(L, G, n, \phi, f, \alpha) \varepsilon .
\end{aligned}
$$

Следовательно, $(L f, \phi)=0$, и $f$ - обобщенное решение уравнения $L u=0$ в $G$.

Установим теперь необходимость условия теоремы 1.

Пусть $\operatorname{mes}_{g} E>0$. Выделяя, в случае необходимости, подмножество, можно считать, что $E$ - компакт. По лемме 2 существует такая борелевская мера $\mu$ с носителем на $E$, что $\mu(E)>0$ и $\mu(B(x, r)) \leqslant g(r)$ для каждого шара $B(x, r) \subset \mathbb{R}^{n}$.

Из леммы 3 , ограниченности области $G$ и неравенства Гёльдера следует, что функционал $\phi \rightarrow \int \phi d \mu$ продолжается по непрерывности с $C_{0}^{\infty}(G)$ до ограниченного функционала на $W_{0}^{1,2}(G)$ (далее при $\phi \in W_{0}^{1,2}(G)$ интеграл $\int \phi d \mu$ понимается как значение этого функционала), поэтому неоднородная задача Дирихле $L f=\mu$ (т.е.

$$
(L f, \phi)=\int \phi d \mu
$$

$\left.\forall \phi \in W_{0}^{1,2}(G)\right)$ разрешима в пространстве $W_{0}^{1,2}(G)$ (доказательство этого факта почти дословно повторяет доказательство теоремы 8.3 из [23]).

Пусть $B(y, r)$ - произвольньй шар, лежаший со своим замыканием в $G$. Положим в равенстве $(5) \phi=f-f_{L, y, r}$. Поскольку $f_{L, y, r}$ - обобщенное решение уравнения $L u=0$ в $B(y, r)$, то

$$
\left(L\left(f-f_{L, y, r}\right), f-f_{L, y, r}\right)=\int\left(f-f_{L, y, r}\right) d \mu .
$$

Оценим по модулю снизу левую часть равенства (6). Имеем

$$
\begin{aligned}
\mid(L(f- & \left.\left.f_{L, y, r}\right), f-f_{L, y, r}\right) \mid \\
= & \mid \sum_{i, j=1}^{n} \int_{B(y, r)} a_{i j} D_{i}\left(f-f_{L, y, r}\right) D_{j}\left(f-f_{L, y, r}\right) d x \\
& +\sum_{i=1}^{n} \int_{B(y, r)} b_{i}\left(f-f_{L, y, r}\right) D_{i}\left(f-f_{L, y, r}\right) d x \\
& \quad-\sum_{i=1}^{n} \int_{B(y, r)} c_{i} D_{i}\left(f-f_{L, y, r}\right)\left(f-f_{L, y, r}\right) d x \\
& -\int_{B(y, r)} d\left(f-f_{L, y, r}\right)\left(f-f_{L, y, r}\right) d x \mid .
\end{aligned}
$$

Пользуясь ограниченностью коэффициентов и равномерной эллиптичностью оператоpa $L$, получаем двойное неравенство

$$
\begin{aligned}
& \lambda \int_{B(y, r)}\left|\nabla\left(f-f_{L, y, r}\right)\right|^{2} d x \\
& \quad \leqslant\left|\sum_{i, j=1}^{n} \int_{B(y, r)} a_{i j} D_{i}\left(f-f_{L, y, r}\right) D_{j}\left(f-f_{L, y, r}\right) d x\right| \\
& \quad \leqslant n^{2} M \int_{B(y, r)}\left|\nabla\left(f-f_{L, y, r}\right)\right|^{2} d x .
\end{aligned}
$$


Вторую сумму в правой части равенства (7) оценим по модулю сверху с помощью неравенств Гёльдера и Пуанкаре

$$
\begin{aligned}
& \left|\sum_{i=1}^{n} \int_{B(y, r)} b_{i}(x)\left(f-f_{L, y, r}\right) D_{i}\left(f-f_{L, y, r}\right) d x\right| \\
& \quad \leqslant n M\left(\int_{B(y, r)}\left|f-f_{L, y, r}\right|^{2}\right)^{1 / 2}\left(\int_{B(y, r)}\left|\nabla\left(f-f_{L, y, r}\right)\right|^{2}\right)^{1 / 2} \\
& \quad \leqslant n M C r\left(\int_{B(y, r)} \mid \nabla\left(f-\left.f_{L, y, r}\right|^{2}\right) d x\right.
\end{aligned}
$$

где $C$ - постоянная в неравенстве Пуанкаре.

Точно также оценивается третья сумма в правой части равенства (7), а последний интеграл в этом равенстве с помощю неравенства Пуанкаре оценивается по модулю сверху величиной $M C^{2} r^{2}$. Поэтому существует такое $r_{0}>0$, зависящее только от $\lambda, M$ и $n$, что при всех $r<r_{0}$ вьполняется неравенство

$$
\frac{\lambda}{2} \int_{B(y, r)}\left|\nabla\left(f-f_{L, y, r}\right)\right|^{2} d x \leqslant\left|\left(L\left(f-f_{L, y, r}\right), f-f_{L, y, r}\right)\right| .
$$

Оценим сверху модуль правой части (6), применяя лемму 3 к сужению меры $\mu$ на шар $B(y, r)$. Имеем

$$
\begin{aligned}
\left|\int_{B(y, r)}\left(f-f_{L, y, r}\right) d \mu(x)\right| & \leqslant K h(r) \| \nabla\left(f-f_{L, y, r}\right) \mid L_{1}(B(y, r) \| \\
& \leqslant C(n) h(r) r^{n / 2} \| \nabla\left(f-f_{L, y, r}\right) \mid L_{2}(B(y, r) \| .
\end{aligned}
$$

Поэтому из (6), (9) и (10) при $r<r_{0}$ получаем

$$
\frac{\lambda}{2}\left\|\nabla\left(f-f_{L, y, r}\right)\left|L_{2}(B(y, r))\left\|^{2} \leqslant C(n) h(r) r^{n / 2}\right\| \nabla\left(f-f_{L, y, r}\right)\right| L_{2}(B(y, r) \|,\right.
$$

откуда

$$
\| \nabla\left(f-f_{L, y, r}\right) \mid L_{2}\left(B(x, r) \| \leqslant 2 C(n) \lambda^{-1} h(r) r^{n / 2}\right.
$$

и, следовательно, $f \in W(h, L, G)$. Теорема доказана.

СледСТВИЕ 1. Пусть $0 \leqslant \alpha \leqslant 1$. Тогда для L-устранимости множества $Е \boldsymbol{в}$ классе $W^{\alpha}(L, G)$ необходимо и достаточно, чтобы хаусдорфова длина Е порядка $n-1+\alpha$ равнялась нулю.

ЗАмЕчаниЕ 1 . Теорема 1 и следствие 1 остаются справедливыми, если в них заме-

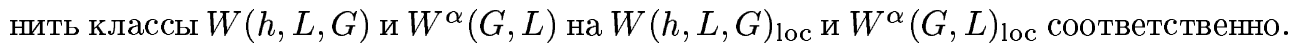

ЗАмЕЧАнИЕ 2. Если $0<\alpha<1$ и $L=\Delta$, то из результатов работы [17] вытекает

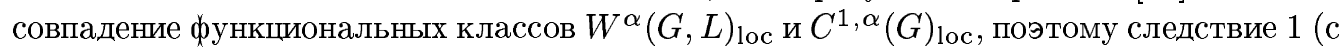
учетом предыдущего замечания) содержит в себе вьшеупомянутьй результат ДолженKO. 
ЗАмЕчАниЕ 3. При доказательстве теоремы 1 фактически установлено, что при $\underline{\lim }_{t \rightarrow 0} h(t) t^{-1}=0$ класс $W(h, L, G)$ состоит только из обобщенных решений уравнения $L u=0$.

ЗАмЕчаниЕ 4. Предположим, что условие (3) не выполнено. В этом случае задача Дирихле, с помощью которой мы определили классы $W(h, L, G)$, может, вообще говоря, быть неразрешимой (см., например, [29, с. 326, 327]). Однако она всегда разрешима на шарах радиуса $r<r_{0}$, где $r_{0}>0$ зависит только от оператора $L$ и области $G$ (и, в более общем случае, в областях достаточно малой лебеговой меры, см. [23], [30]). Поэтому определение классов $W(h, L, G)$ сохраняется со следующей модификацией: если задача Дирихле $L u=0, u-f \in W_{0}^{1,2}(B(x, r))$ в шаре $B(x, r)$ однозначно разрешима, то $f_{L, x, r}-$ ее решение; в противном случае $f_{L, x, r} \equiv f$ в $B(x, r)$. Теорема 1 в этом случае остается в силе, если ограничиться рассмотрением компактных множеств особых точек и определить понятие устранимости множества $E$ по аналогии с тем, как это обычно делается в случае задачи Пенлеве (характеризации компактных множеств, устранимых для класса ограниченных аналитических функций). А именно, будем назьвать компакт $E \subset G$ $(L, h)$-устранимым, если для любой области $G_{1} \subset G$ такой, что $E \subset G_{1}$, и для любой функции $f \in W\left(h, L, G_{1}\right)$, которая в $G_{1} \backslash E$ является обобщенным решением уравнения $L u=0$, вьполняется следующее: функция $f$ после, возможно, переопределения ее на $E$ становится обобшенньм решением уравнения $L u=0$ в $G_{1}$.

ЗАмЕчАниЕ 5. В последние годы интенсивно изучаются решения вырождающихся квазилинейных эллиптических уравнений (см., например, монографию [31]), в частности, так назьваемые $p$-гармонические функции. Пусть $1<p<\infty$. Напомним, что функция $f \in W^{1, p}(G)$ lос называется $p$-гармонической в области $G$, если для любой функции $\phi \in C_{0}^{\infty}(G)$ выполняется равенство

$$
\int_{G}|\nabla f|^{p-2} \nabla f \cdot \nabla \phi d x=0
$$

(точкой обозначено скалярное произведение векторов; если $p<2$ и $\nabla f=0$, то считаем $\left.|\nabla f|^{p-2} \nabla f=0\right)$. Каждая такая функция после, возможно, переопределения на мно-

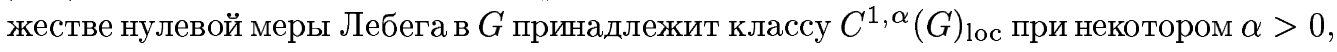
зависящем лиш от $n$ и $p$, и после такого переопределения становится бесконечно дифференцируемой на множестве $\{x \in G: \nabla f(x) \neq 0\}$. В работе [32] доказан следуюший результат об устранимости замкнутых множеств $E \subset G, E \neq G$, для $p$-гармонических функций в классе $C^{\alpha}(G)_{\text {loc }}, 0<\alpha<1$ : множество $E$ устранимо тогда и только тогда, когда хаусдорфова длина $E$ порядка $n-p+\alpha(p-1)$ равна нулю. Пусть $q=p /(p-1)$ и $h-$ такая же функция, как и выше. Рассмотрим множество $U_{p}(h, G)$ (которое, вообще говоря, не является линейным пространством) всех функций $f \in W^{1, p}(G)_{\text {lос }}$, удовлетворяющих следующему условию: для каждого шара $B(x, r)$, лежашего со своим замыканием в $G$, и для каждой функции $\phi \in C_{0}^{\infty}(B(x, r))$ вьполняется неравенство

$$
\left.\left|\int_{B(x, r)}\right| \nabla f\right|^{p-2} \nabla f \cdot \nabla \phi d x\left|\leqslant C h(r) r^{n / q}\left\|\nabla \phi \mid L_{p}(B(x, r))\right\|,\right.
$$

где $C \geqslant 0$ не зависит от $r$ и функции $\phi$. Тогда, фактически повторяя доказательство теоремы 1 (комментария здесь требует только доказательство существования функции 
$f \in W_{0}^{1, p}(G)$ такой, что для любой $\phi \in C_{0}^{\infty}(G)$ выполняется равенство

$$
\int_{G}|\nabla f|^{p-2} \nabla f \cdot \nabla \phi d x=\int_{G} \phi d \mu,
$$

где $\mu$ - та же мера, что и вьше; это установлено, например, в [33], см. также [34, с. 204]), мы получаем, что ее условие является необходимьм и достаточньм для устранимости множества $E$ для $p$-гармонических функций в классе $U_{p}(h, G)$ (устранимость здесь понимается так же, как и в определении 1). При $p=2$ этот класс совпадает с классом $W(h, \Delta, G)$. В частности, при $h(t)=t^{\alpha}, 0<\alpha<1$, класс $U_{2}(h, G)_{\text {lос совпада- }}$ ет с классом $C^{1, \alpha}(G)_{\text {loc }}$, и сформулированньй результат может рассматриваться как обобщение теоремы Долженко на случай $p$-гармонических функций. С естественными изменениями сказанное в этом замечании переносится на решения более общих вырождающихся квазилинейных эллиптических уравнений, рассматриваемых в [31].

Основной результат работы впервые доложен на Международной конференции по дифференциальным уравнениям и динамическим системам в Суздале в июле 2002 г. (см. [35]).

\section{СПИСОК ЦИТИРОВАННОЙ ЛИТЕРАТУРЫ}

[1] Долженко Е.П. О представлении непрерывных гармонических функций в виде потенциалов // Изв. АН СССР. Сер. матем. 1964. Т. 28. № 5. С. 1113-1130.

[2] Долженко Е.П. Об особых точках непрерьвных гармонических функций // Изв. АН CССР. Сер. матем. 1964. Т. 28. №6. С. 1251-1270.

[3] Carleson L. Removable singularities for continuous harmonic functions in $\mathbb{R}^{n} / /$ Math. Scand. 1963. V. 12. P. 15-18.

[4] Карлесон Л. Избранные проблемы теории исключительных множеств. М.: Мир, 1971.

[5] Harvey R., Polking J. C. Removable singularities of solutions of linear partial differntial equations // Acta Math. 1970. V. 125. №1/2. P. 39-56.

[6] Kral J. Removable singularities of solutions of semielliptic equations // Rendiconti de Matematica. 1973. V. 6. № 4. P. 763-783.

[7] Kral J. Semielliptic singularities // Casopis pro pest. mat. 1984. V. 109. P. 204-322.

[8] Ищанов Б. ЖК. Об устранимых особенностях функций классов $B M O$ и их обобщений // Вестн. Моск. ун-та. Сер. 1. Матем., мех. 1985. № 5. С. 77-80.

[9] Ищанов Б. ЖК. Незамкнутые особые множества для слабых решений линейньх дифференциальных уравнений // Геометрические вопросы теории функций и множеств. Калинин: Калининский гос. ун-т, 1989. С. 41-49.

[10] Ищанов Б. Ж . О представлении решений некоторых классов уравнений в виде потенциалов мер, распределенных на множестве особенностей // Докл. РАН. 1998. Т. 358. № 4. С. 455-458.

[11] Uy N.X. Removable set for Lipschitz harmonic functions // Michigan Math. J. 1990. V. 37. P. 45-51.

[12] Verdera J. $C^{m}$-approximations by solutions of elliptic equations and Calderon-Zygmund operators // Duke Math. J. 1987. V. 55. № 1. P. 157-187.

[13] Mateu J., Orobitg J. Lipschitz approximations by harmonic functions // Indiana Univ. Math. J. 1990. V. 39. P. 703-736.

[14] Ullrich D. C. Removable sets for harmonic functions // Michigan Math. J. 1991. V. 38. №3. P. 467-473.

[15] Тарханов Н.Н. Ряд Лорана для решений эллиптических систем. Новосибирск: Наука, 1991.

[16] David G., Mattila P. Removable sets for Lipschitz harmonic functions in the plane // Revista Mat. Iberoamericana. 2000. V. 16. P. 137-215.

[17] Покровский А. В. Локальные аппроксимации решениями гипоэллиптических уравнений и устранимые особенности // Докл. РАН. 1999. Т. 367. №1. С. 15-17. 
[18] Покровский А.В. Об устранимых особенностях решений однородных эллиптических уравнений в классах Никольского-Бесова // Докл. РАН. 2001. Т. 380. № 2. С. 168-171.

[19] Кондратьев В.А., Ландис Е. М. Качественная теория линейных дифференциальных уравнений в частных производных второго порядка // Итоги науки и техники. Современные проблемы математики. Фундаментальные направления. Т. 32. М.: ВИНИТИ, 1988. С. 99-215.

[20] De Giorgi E. Sulla differenziabilita e l'analiticita delle estremali degli integrali multipli regolari // Mem. Acad. Sci. Torino. Ser. 3. 1957. № 1. P. 25-38.

[21] Nash J. Continuity of solutions of parabolic and elliptic equations // Amer. J. Math. 1958. V. 80. P. 931-954.

[22] Ландис Е. М. Уравнения второго порядка эллиптического и параболического типа. М.: Наука, 1971.

[23] Гилбарг Д., Трудингер Н. Эллиптические дифференциальные уравнения с частными производными второго порядка. М.: Наука, 1989.

[24] Фёдоров B. C. Sur la representatione des fonctions analitiques au voisinage d'un ensemble de ses points singuliers // Матем. сб. 1928. Т. 35. С. 237-250.

[25] Долженко Е. П. Работы Д. Е. Меньшова по теории аналитических функций и современное состояние теории моногенности // УМН. 1992. Т. 47. № 5(287). С. 67-96.

[26] Frostman O. Potentiel d'équilibre et capacité des ensembles avec quelques applications à la théorie de fonctions // Meddel. Lunds Univ. Mat. Sem. 1935. V. 3. P. 1-118.

[27] Хейман У., Кеннеди П. Субгармонические функции. М.: Мир, 1980.

[28] Мазья В. Г. Пространства С. Л. Соболева. Л.: Изд. Ленинград. ун-та, 1985.

[29] Петровский И. Г. Лекции об уравнениях с частными производными. М.: ГИФМЛ, 1961.

[30] Кондратьев В.А. О разрешимости первой краевой задачи для сильн эллиптических уравнений // Тр. ММО. 19???. Т. 16. С. 293-318.

[31] Heinonen J., Kilpeläinen T., Martio O. Nonlinear Potential Theory of Degenerate Elliptic Equations. Oxford: Oxford Univ. Press, 1993.

[32] Kilpeläinen T., Zhong X. Removable sets for continuous solutions of quasilinear elliptic equations // Proc. Amer. Math. Soc. 2002. V. 130. №6. P. 1681-1688.

[33] Мазья В.Г. О непрерывности в граничной точке решений квазилинейных эллиптических уравнений // Вестн. Ленинград. ун-та. 1970. №13. С. 42-55.

[34] Куфнер А., Фучик С. Нелинейные дифференциальные уравнения. М.: Наука, 1988.

[35] Pokrovskii A. V. Removable singularities for solutions to second-order elliptic equations // International Conference on Differential Equations and Dynamical Systems (Suzdal, July 1-6, 2002). Abstracts. Vladimir, 2002. P. 195-196.

Институт математики НАН Украины, г. Киев

Поступило

E-mail: pokrovsk@math.kiev.ua

17.10 .2003 